\title{
The Raman spectrum of isolated water clusters $\dagger$
}

Cite this: Phys. Chem. Chem. Phys., 2014, 16, 9849

Received 10th October 2013, Accepted 29th November 2013 DOI: $10.1039 / c 3 c p 54272 f$

www.rsc.org/pccp

\author{
Katharina E. Otto, Zhifeng Xue, Philipp Zielke and Martin A. Suhm*
}

Cold water oligomers $\left(\mathrm{H}_{2} \mathrm{O}\right)_{n}$ and $\left(\mathrm{D}_{2} \mathrm{O}\right)_{n}$ with $n=2-5$ are assigned in spontaneous Raman scattering spectra of seeded rare gas expansions for the first time. Comparison with infrared spectra provides direct experimental insights into the hydrogen bond-mediated excitonic $\mathrm{OH}$ oscillator coupling, which is responsible for ultrafast energy transfer between water molecules, usually suppressed by isotopic dilution in femtosecond experiments for the condensed phase. The experimental coupling constants are compared to those in state-of-the-art full-dimensional water potential energy hypersurfaces, leaving room for improvement in the description of the coupled dynamics in water. Evidence for intensified Fermi resonance between $\mathrm{OH}$ stretching and $\mathrm{OH}$ bending motion beyond water trimers is collected.

\section{Introduction}

The vibrational dynamics of water is of fundamental interest. ${ }^{1}$ It is mediated by a network of intermolecular hydrogen bonds, which can be introduced step by step in the popular cluster approach. ${ }^{2,3}$ While matrix isolation ${ }^{4,5}$ and chromophore labeling ${ }^{6,7}$ have proven useful in this context, the study of unperturbed, isolated water clusters is particularly attractive due to the close contact with theory ${ }^{8}$ and the large amplitude motion in these systems. Direct infrared ${ }^{2,9-11}$ and microwave ${ }^{12}$ studies have become available in recent years and have provided detailed information on nuclear quantum effects and electronic cooperativity, in particular when combined with theoretical predictions. Although the Raman selection rules provide essential complementary information on concerted motions, only a pioneering coherent anti-Stokes Raman spectroscopy (CARS) investigation of isolated water clusters has so far been published ${ }^{13}$ and discussed controversially. ${ }^{14,15}$ The nonlinearity of the CARS experiment rendered a firm assignment of cluster sizes difficult. Therefore, the characteristic coupling between neighboring $\mathrm{OH}$ oscillators in water assemblies, which controls energy flow after local excitation, has remained largely in the dark.

This is where the present contribution sets in. By generating isolated, internally cold, small water clusters in slit jet expansions of an atomic carrier gas into vacuum and probing them

Institut für Physikalische Chemie, Georg-August-Universität Göttingen, Tammannstraße 6, 37077 Göttingen, Germany.E-mail: msuhm@gwdg.de; Fax: +49 551 3933117; Tel: +49 5513933112

$\dagger$ Electronic supplementary information (ESI) available: Rotational temperature analysis based on Stokes and anti-Stokes intensities; detailed descriptions of spectra and experimental conditions; depolarization analysis; matrix isolation trends; and more extensive quantum chemical compilations. See DOI: 10.1039/ c3cp54272f by an intense visible laser, sufficient numbers of spontaneously Raman-scattered photons are generated to detect $\mathrm{OH}$ stretching modes which show negligible infrared intensity and have thus remained unassigned in the gas phase to date. The strong hydrogen bond cooperativity ensures a sufficient spread of the signals on the wavenumber axis for a reliable cluster size separation and size assignment based on stagnation pressure, nozzle temperature, polarization dependence ${ }^{16}$ and water concentration dependence up to at least five water units. Further support for the vibrational and size assignment comes from equivalent experiments with $\mathrm{D}_{2} \mathrm{O}$. This compensates for the lack of rigorous size selection. ${ }^{3,17}$ The combination with corresponding IR data ${ }^{10,18}$ then leads to an effective approach to the oscillator coupling within a simple framework of Hückellike coupling matrices, ${ }^{7}$ as exemplified in the case of cyclic methanol clusters. ${ }^{19}$ The energy splitting of the degenerate monomer oscillators into symmetric and asymmetric linear combinations in a cyclic cluster is caused by coupling through space or through the connecting hydrogen bonds.

These coupled modes are observable as a mode pattern in the frequency domain, ${ }^{20}$ but their importance is better visualized in the time domain. Excitation of a local $\mathrm{OH}$ stretching mode by a suitable ultrashort laser pulse would lead to a rapid delocalization of the excitation energy among the coupled oscillators as a function of time. In a finite cluster and in the absence of other redistribution mechanisms, this is a periodic process which ultimately leads to a refocussing of the energy in the original oscillator. In liquid or solid water, the energy is dissipated irreversibly. Although the detailed quantum dynamics can be complex, there is a reciprocal relationship between the magnitude of the excitonic splitting and the lifetime of the localized state. The larger the spectral splitting, the faster the energy dissipation. Therefore, the size of the coupling constant between $\mathrm{OH}$ stretching modes in a water cluster is of 
prime importance for the femtosecond dynamics of these high frequency oscillators. ${ }^{16,21,22}$ Because the ultrafast exciton dynamics among degenerate oscillators leads to rapid anisotropy decay, most femtosecond experiments and simulations on liquid water are carried out in isotopic dilution to focus on slower processes. ${ }^{1,23,24}$ In liquid water, the effective excitonic coupling constant has been estimated ${ }^{25}$ to be around $12 \mathrm{~cm}^{-1}$. In cold ring-like clusters, it is modelled to be of the same order of magnitude for the bonded $\mathrm{OH}$ stretch mode. ${ }^{26}$ This is significantly smaller than in the related alcohol clusters ${ }^{19}$ (20-30 $\mathrm{cm}^{-1}$ ), which feature the same hydrogen bonded ring topology. ${ }^{27}$ We will argue in this contribution that the experimental coupling constants in ring-like water clusters are indeed larger than analytically modelled and more in line with those observed in methanol clusters. The associated concerted stretching modes of water ring clusters are particularly interesting and important because they represent a low energy window of the potential energy hypersurface where water molecules lose their integrity and start to undergo isotope exchange. ${ }^{28}$ These modes are also predicted to have exceptionally fast IVR rates. ${ }^{29}$

One important aspect has to be considered in the $\mathrm{OH}$ stretch coupling of small water clusters. In isolated water molecules, the $\mathrm{OH}$ bending overtone is located more than $500 \mathrm{~cm}^{-1}$ below the symmetric stretching fundamental ${ }^{30}$ but it comes into resonance for cooperatively weakened $\mathrm{OH}$ bonds. As we will argue based on Raman spectroscopic evidence, this hydrogen bond-tuned Fermi resonance becomes increasingly important when moving from the trimer to the pentamer, opening up another energy flow channel in particular for symmetric and thus Raman-active excitation. The corresponding normal modes are visualized for the pentamer case in Fig. 1.

While the focus of this contribution is on cyclic trimers, tetramers and pentamers with their low microwave visibility ${ }^{12}$ but well-characterized low-frequency dynamics, ${ }^{2,31}$ we also present the first Raman spectra for isolated water dimers, for which some transitions have previously been observed in $\mathrm{He}$ nanodroplets. ${ }^{32}$ Finally, we provide circumstantial evidence for the topologically versatile hexamer. ${ }^{2,12,33-36}$

After a brief description of the experimental setup, the spontaneous Raman spectra will be presented and assigned. The discussion of excitonic patterns in the cyclic clusters will start with the most clearcut tetramer case and extend to the

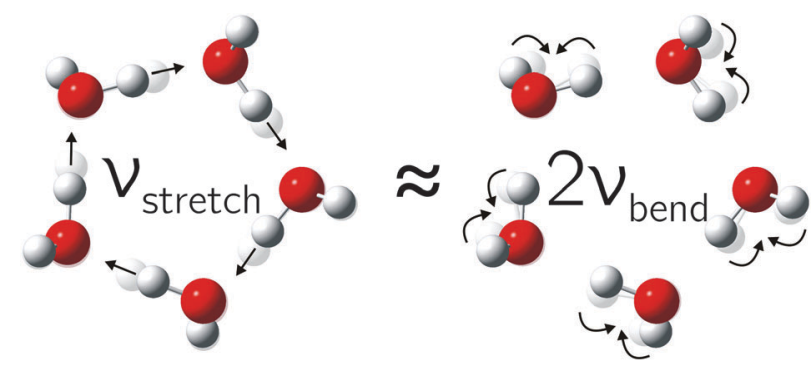

Fig. 1 The in-phase hydrogen-bonded $\mathrm{OH}$ stretching fundamental is closely resonant with the overtone of the highest frequency bending mode in the water pentamer (and less so in the tetramer). trimer and pentamer, followed by preliminary assignments of non-cyclic hexamer bands.

\section{Experimental setup}

Gas mixtures of water with $\mathrm{He}, \mathrm{Ne} / \mathrm{He}$ or $\mathrm{Ar} / \mathrm{He}$ were expanded via a $4 \times 0.15 \mathrm{~mm}^{2}$ or $8 \times 0.05 \mathrm{~mm}^{2}$ heatable slit nozzle into an aluminium chamber, pumped using two Roots pumps $\left(500 \mathrm{~m}^{3} \mathrm{~h}^{-1}\right.$ and $\left.250 \mathrm{~m}^{3} \mathrm{~h}^{-1}\right)$ and one rotary vane pump $\left(100 \mathrm{~m}^{3} \mathrm{~h}^{-1}\right)$. The beam of a frequency doubled cw $\mathrm{NdYVO}_{4}$-Laser (Coherent Verdi $\mathrm{V} 18,18 \mathrm{~W}, \lambda=532 \mathrm{~nm}$ ) was focused onto the expansion at $2 \mathrm{~mm}$ distance from the nozzle exit. The scattered light was collected perpendicular to the expansion and to the incident laser beam and collimated using a fast camera lens (50 mm Nikon, $f / 1.2$ ). It was then focused onto the entrance slit of the monochromator (McPherson Model 2051 $f / 8.6, f=1000 \mathrm{~mm}$, grating 1200 grooves per $\mathrm{mm}$ or McPherson Model $205 \mathrm{f} f / 3.2, f=500 \mathrm{~mm}$, grating 600 gr per $\mathrm{mm}$ ) using an achromatic planoconvex lens (Edmund Optics, $\varnothing=50 \mathrm{~mm}, \mathrm{f} / 7$ for McPherson Model 2051 and Edmund Optics, $\varnothing=50 \mathrm{~mm}, f / 4$ for McPherson Model 205f). Suppression of the Rayleigh scattered light was achieved using a Raman edge filter (L.O.T., $\varnothing=25 \mathrm{~mm}$, OD 6.0, $T>$ 90\%, 535.4-1200 nm). A back-illuminated CCD camera (PI Acton, Spec-10: $400 \mathrm{~B} / \mathrm{LN}, 1340 \times 400$ pixels, cooled by liquid $\mathrm{N}_{2}$ ) served for detection in a vertical binning mode. ${ }^{19}$ Count rates refer to the full 400-pixel columns. The wavelength calibration of the spectra was carried out using the lines of a Ne I emission light source and $\mathrm{H}_{2} \mathrm{O}$ monomer Raman transitions. ${ }^{30,37}$ Cosmic ray signals were removed by the comparison of block-averaged spectra. Polarization experiments were performed by rotating the laser beam via a $\lambda / 2$-plate.

The Stokes scattering was probed from 80 to $8000 \mathrm{~cm}^{-1}$, but here we concentrate on the by far strongest ${ }^{38}$ Raman cluster signals between 3000 and $4000 \mathrm{~cm}^{-1}$, in the region of the $\mathrm{OH}$ stretching fundamentals and the $\mathrm{OH}$ bending overtones.

\section{Results and discussion}

\subsection{Spectral survey}

In supersonic jet expansions, different molecular degrees of freedom may have different effective temperatures or even nonthermal distributions. We obtained monomer rotational temperatures of 30-50 K based on the relative intensities of Stokes and anti-Stokes transitions (see ESI $\dagger$ for details). These are in qualitative agreement with recent results for cluster-free expansions of water in helium, considering the large differences in expansion conditions. ${ }^{37}$ Vibrational temperatures are probably higher and lead to relatively broad band contours with occasional hot band features, marked with * in the following spectra. The temperatures can be lowered by variation of the stagnation conditions and carrier gas.

Fig. 2 and 3 contain a collection of Raman supersonic jet spectra for $\mathrm{H}_{2} \mathrm{O}$ and $\mathrm{D}_{2} \mathrm{O}$ expansions in different carrier gases and under varying conditions (described in detail in the ESI $\dagger$ ). Their spectral windows have been matched by $\mathrm{D}_{2} \mathrm{O}$ wavenumber 


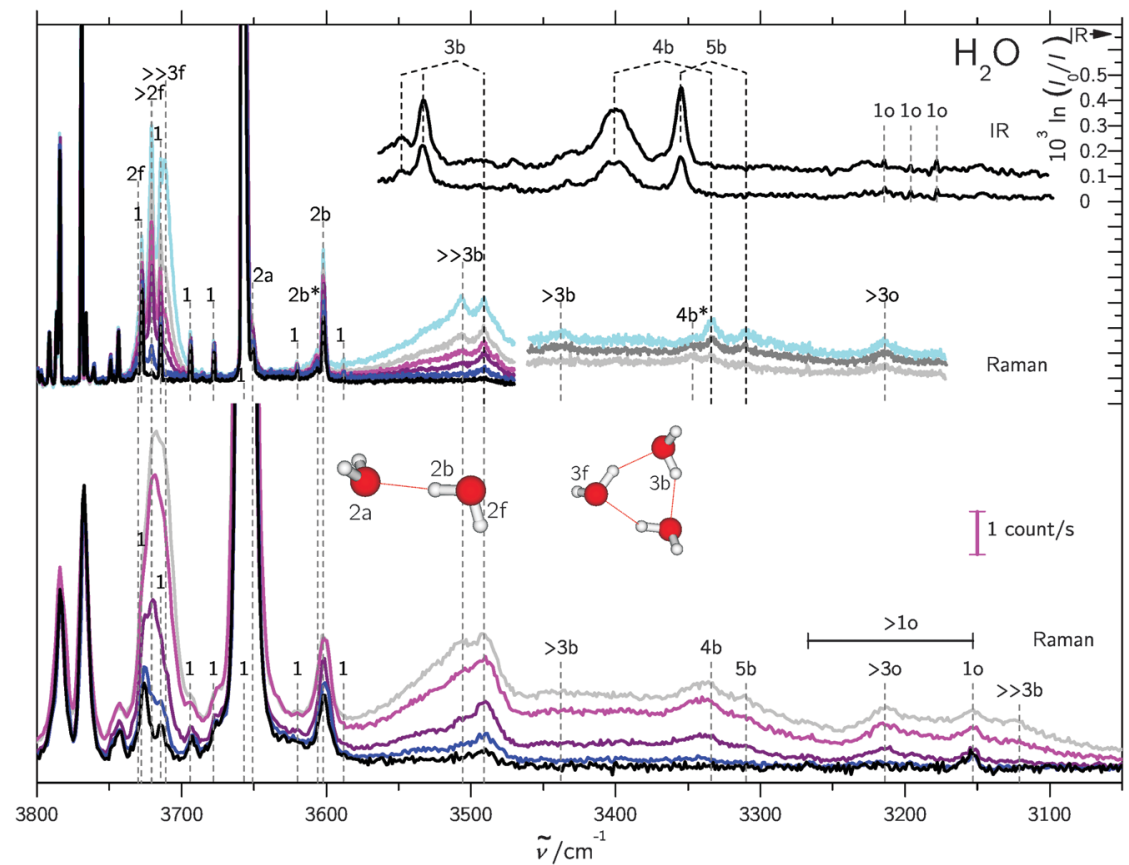

Fig. 2 Raman and $I \mathrm{R}^{10}$ spectra of mixed $\mathrm{H}_{2} \mathrm{O} /$ rare gas expansions. Raman spectra were scaled to the same monomer scattering intensity at the point of measurement in the respective blocks. The labels are defined in the text and in Table 1. Detailed information on the measurement conditions, such as nozzle temperature $\vartheta_{n}$, saturator temperature $\vartheta_{\mathrm{s}}$ and stagnation pressure $p_{\mathrm{s}}$ can be found in Table SI in the ESI. $\dagger$ Lower part: Raman spectra recorded using a $0.5 \mathrm{~m}$ monochromator. Basically, the clustering extent increases from bottom to top. Upper part: similar conditions but recorded using a $1 \mathrm{~m}$ monochromator. Again, the clustering extent increases from bottom to top.

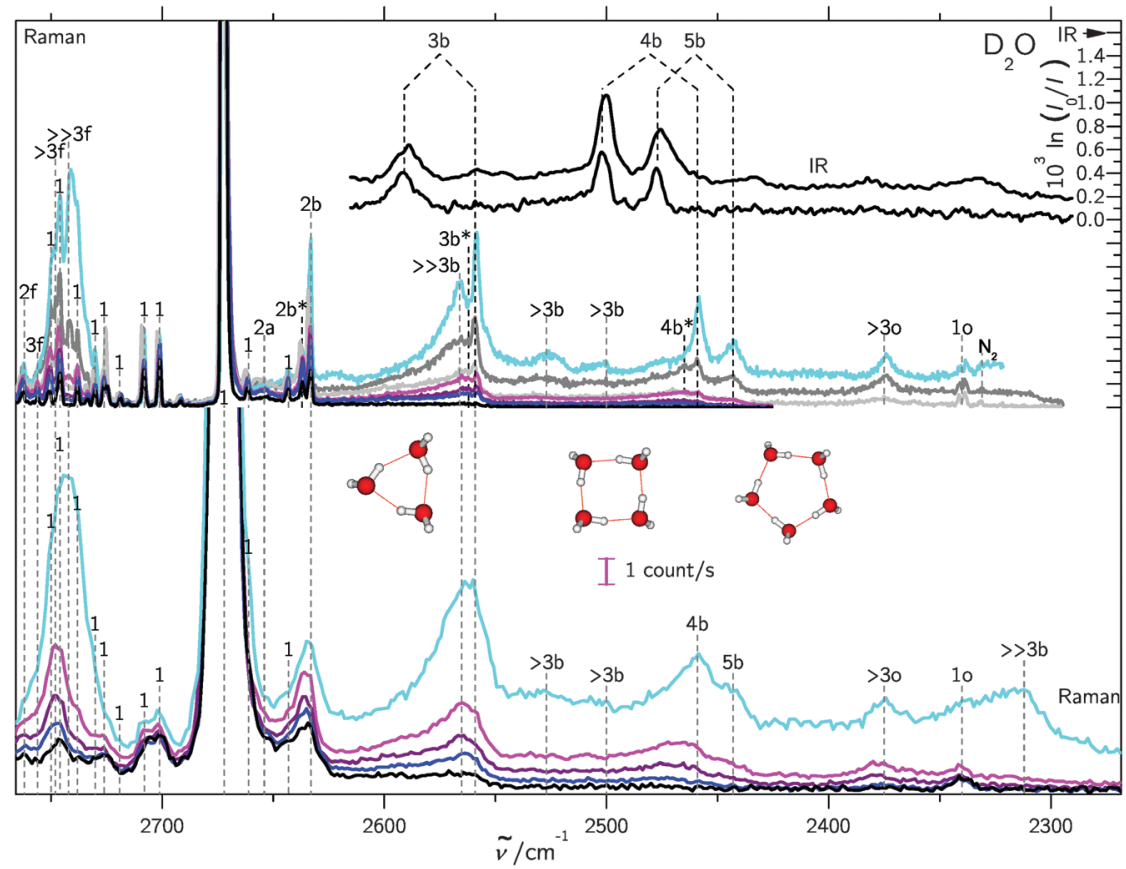

Fig. 3 Like Fig. 2, but for $\mathrm{D}_{2} \mathrm{O} /$ rare gas expansions, also showing trimer to pentamer structures. For detailed information see Table SII in the ESI. $\dagger$

axis stretching $(\times 1.508)$ and monomer symmetric stretch band center alignment. The correspondence between the isotopologues is indeed very close. Studies of the carrier gas dependence and of the depolarization ratio are provided in the ESI. $\uparrow$ Table 1 provides peak positions and explains the labels of the Raman spectral features. Also shown in the upper right traces and in Table 1 are corresponding FTIR supersonic jet spectra, ${ }^{10}$ which agree with earlier laser spectroscopy observations. ${ }^{18,39}$

The high degree of complementarity for trimers (3b), tetramers (4b) and pentamers (5b) is a consequence of their ring topology. 
Table 1 Important experimental peak positions in $\mathrm{H}_{2} \mathrm{O}$ and $\mathrm{D}_{2} \mathrm{O}$ expansions and their tentative assignments. The number stands for the cluster size, $\mathrm{b}$ for bound $\mathrm{OH} \cdots \mathrm{O}, \mathrm{f}$ for free $\mathrm{OH}$, a for acceptor, o for bending overtone, and * for a hot band. For the $\mathrm{H}_{2} \mathrm{O}$ monomer overtone transitions also the rotational transition quantum numbers in the order $J, K_{a}, K_{c}$ for the final and initial levels are given

\begin{tabular}{lll}
\hline Peak position $/ \mathrm{cm}^{-1}$ & & \\
\hline $\mathrm{H}_{2} \mathrm{O}$ & $\mathrm{D}_{2} \mathrm{O}$ & Assignment \\
\hline 3730 & 2762 & $2 \mathrm{f}$ \\
3721 & - & $>2 \mathrm{f}$ \\
- & 2756 & $3 \mathrm{f}$ \\
- & 2748 & $>3 \mathrm{f}$ \\
3711 & 2742 & $\gg 3 \mathrm{f}$ \\
3657 & 2672 & 1 \\
3651 & 2654 & $2 \mathrm{a}$ \\
3606 & 2637 & $2 \mathrm{~b}^{*}$ \\
3602 & 2633 & $2 \mathrm{~b}$ \\
3548 & - & $3 \mathrm{~b}(\mathrm{IR}-\mathrm{w})$ \\
3533 & 2591 & $3 \mathrm{~b}(\mathrm{IR})$ \\
3506 & 2566 & $\gg 3 \mathrm{~b}$ \\
- & 2562 & $3 \mathrm{~b}^{*}$ \\
3491 & 2559 & $3 \mathrm{~b}$ \\
3438 & 2527 & $>3 \mathrm{~b}(4 \mathrm{~b} ?)$ \\
3401 & 2502 & $4 \mathrm{~b}(\mathrm{IR})$ \\
- & 2500 & $>3 \mathrm{~b}(5 \mathrm{~b} ?)$ \\
3355 & 2477 & $5 \mathrm{~b}(\mathrm{IR})$ \\
3347 & 2465 & $4 \mathrm{~b}^{*}$ \\
3334 & 2459 & $4 \mathrm{~b}$ \\
3310 & 2443 & $5 \mathrm{~b}$ \\
3214 & 2375 & $>3 \mathrm{o}$ \\
3215 & - & $1 \mathrm{o}(\mathrm{IR})(212) \leftarrow(101)$ \\
3196 & - & $1 \mathrm{o}(\mathrm{IR})(111) \leftarrow(000)$ \\
3178 & - & $1 \mathrm{o}(\mathrm{IR})(110) \leftarrow(101)$ \\
3153 & 2340 & $1 \mathrm{o}(n 0 n) \leftarrow(n 0 n)$ \\
3121 & 2312 & $\gg 3 \mathrm{~b}$ \\
& &
\end{tabular}

The Raman spectra are dominated by the sharp symmetric $\mathrm{OH}$ stretching transitions of the monomer, some of which are labeled (1). Between the symmetric and antisymmetric water monomer bands, a structured scattering signal from dangling (free) $\mathrm{OH}$ groups is found. Contributions from dimers (2f), trimers (3f, $>2 \mathrm{f})$, larger $(>3 \mathrm{f})$ and significantly larger $(\gg 3 \mathrm{f})$ clusters can be partially separated, but their discussion will be postponed. They are also summarized in Table 1 for both light and heavy water.

A general feature of the warmer Raman spectra is the asymmetric slope of the hydrogen-bonded stretching bands due to thermal excitation. This reflects the sensitivity of the $\mathrm{OH}$ stretching wavenumber to the strength of the hydrogen bond, so important in fs bulk water experiments ${ }^{1}$ and in the room temperature detection of water clusters. ${ }^{40,41}$ The colder spectra using heavier carrier gases (see ESI $\dagger$ ) are substantially more narrow, but show larger contributions from clusters with a hydrogen bond network $(n>5)$, which are beyond the safe size resolution of our Raman technique.

In the following, we therefore concentrate on the cyclic trimer, tetramer and pentamer Raman features and their interpretation in terms of intermolecular and intramolecular couplings.

\subsection{Water tetramer}

The cyclic tetramer is particularly well suited for an analysis of the $\mathrm{OH}$ coupling patterns derived from the combined IR and Raman spectra ${ }^{19}$ because it has four equivalent hydrogen bonds arranged on the sides of a square. Each $\mathrm{OH}$ group has the same zeroth order wavenumber and couples directly to its hydrogen bond acceptor partner and either along two hydrogen bonds or across the diagonal to its next-nearest neighbor. The direct coupling is described by $W_{1}$ and the weaker diagonal coupling by $W_{2}$. A Hückel-like analysis ${ }^{19}$ of the combined action of all couplings leads to the determinant equation

$$
\left|\begin{array}{cccc}
x & W_{1} & W_{2} & W_{1} \\
W_{1} & x & W_{1} & W_{2} \\
W_{2} & W_{1} & x & W_{1} \\
W_{1} & W_{2} & W_{1} & x
\end{array}\right|=0
$$

the solutions of which are

$$
\begin{gathered}
x_{1}=-2 W_{1}-W_{2} \\
x_{2,3}=W_{2} \\
x_{4}=2 W_{1}-W_{2}
\end{gathered}
$$

The symmetry labels A, E, B derive from the $\mathrm{S}_{4}$ point group. The degenerate pair of $\mathrm{E}$ transitions, which is predominantly IR-active, is thus separated by $W_{2}$ from the center of the coupling pattern, whereas the predominantly Raman-active A transition is below the center, by $2 W_{1}+W_{2}$. This A level was recently predicted to have an intracluster energy redistribution rate of $0.4 \mathrm{ps}^{29}$ The observed width of the Raman transition of $10 \mathrm{~cm}^{-1}$ is qualitatively still compatible with this rate in the limit of a dense manifold of coupling states, but actually more narrow than the corresponding IR mode. The B-transition is expected to have a weak intensity in both types of spectra. The splitting between the IR-active and dominant Raman active bands (E/A) is thus $2 W_{1}+2 W_{2}$. Between the weaker Ramanactive and the IR-active bands $(\mathrm{B} / \mathrm{E})$ it is $2 W_{1}-2 W_{2}$, and between the two Raman active bands (B/A) $4 W_{1}$. As the assignment of the second Raman active band is less certain, we define a composite coupling constant $W 4=W_{1}+W_{2}$ which only depends on the strong Raman and IR transitions (corresponding to one half of the E/A separation) and allows for a safer comparison between theory and experiment. The experimental error of W4 may be conservatively estimated to be around $\pm 2 \mathrm{~cm}^{-1}$ as one half of the sum of the Raman calibration error of $\pm 1 \mathrm{~cm}^{-1}$ and possible differences between band centers and band maxima in the IR and Raman spectra of up to $3 \mathrm{~cm}^{-1}$, which should however largely cancel in the spectral difference.

Depending on the spectral assignments, there are three different ways to analyze the coupling multiplet in the water tetramer. If one neglects couplings beyond the nearest neighbors $\left(W_{2}\right)$, the direct coupling constant based on the most active A-symmetric (Raman) and E-symmetric (IR) vibrations is $W 4 \approx W_{1}=34 \mathrm{~cm}^{-1}$, which is to be compared to $12-13 \mathrm{~cm}^{-1}$ from the most recent and otherwise fairly accurate analytical potential hypersurface. ${ }^{42}$ One could argue that the discrepancy stems from the neglect of next-nearest neighbor coupling. Indeed, inclusion of the weakly Raman-active B-transition at $3438 \mathrm{~cm}^{-1}$ reduces the experimental nearest-neighbor coupling 
Table 2 Different theoretical results for the nearest neighbor $\left(W_{1}\right)$ and distant $\left(W_{2}\right)$ coupling parameters of the hydrogen-bonded OH stretching manifold of the water tetramer compared to the present experimental results for $\left(\mathrm{H}_{2} \mathrm{O}\right)_{4}$ and $\left(\mathrm{D}_{2} \mathrm{O}\right)_{4}$ as well as the more strongly bound "methylated" water tetramers. ${ }^{19}$ The tabulated sum $W 4=W_{1}+W_{2}$ is particularly robust from the experimental point of view

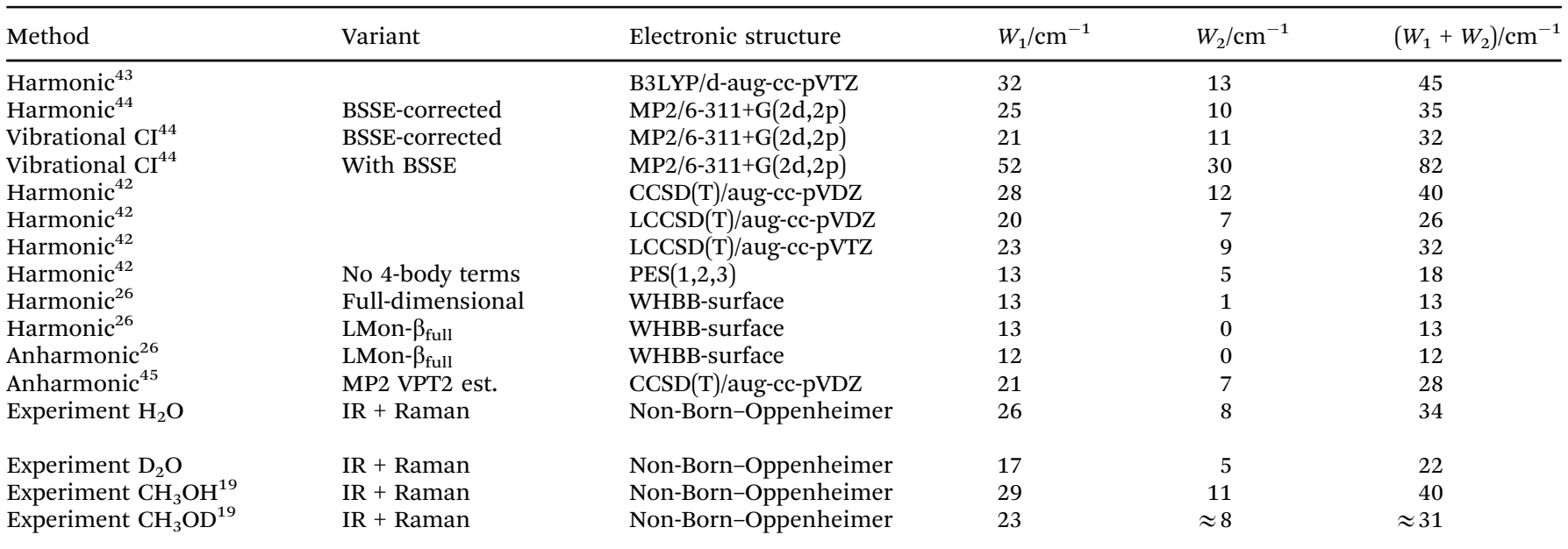

to $W_{1}=26 \mathrm{~cm}^{-1}$ by compensating with a distant coupling term across the ring of $W_{2}=8 \mathrm{~cm}^{-1}$. This does not improve the agreement with theory, because such a second-nearest neighbor interaction is essentially absent from the most recent analytical potential energy hypersurfaces. In contrast, even simple harmonic $a b$ initio calculations yield largely correct orders of magnitude, also in line with experiments for methanol tetramers. ${ }^{19}$ If one does not want to rely on the less certain position of the B-band, one evaluates the sum $W_{1}+W_{2}$, defined above as the B-invariant tetramer coupling $W 4$, which corresponds to one half of the IR/Raman band splitting. It is equivalent to $W_{1}$ only if the diagonal coupling $W_{2}$ is negligible. Table 2 summarizes the experimental findings for the different assumptions and isotopologues and compares them to the methanol findings. Dependent on the isotopic composition and analysis method, the methanol coupling is seen to be 10-60\% larger than the water coupling, in line with qualitative expectations on the strength of the hydrogen bond and susceptible to some perturbations in the $\mathrm{CH}_{3} \mathrm{OD}$ case. ${ }^{19}$ Upon deuteration, the coupling constants shrink by $30-40 \%$, as in methanol. ${ }^{19}$

In view of the involved simplifications, the agreement between experiment and harmonic ab initio calculations is remarkably good for the $\left(\mathrm{H}_{2} \mathrm{O}\right)_{4}$ coupling constants. While the B3LYP calculations ${ }^{43}$ somewhat overestimate the coupling, available BSSE-corrected MP2 and higher level electron correlation treatments ${ }^{42,46}$ agree with experiment within the uncertainties of the latter and vibrational CI treatment does not change the values substantially. ${ }^{46}$ This contrasts with the performance of analytical potential energy surfaces derived from these highly correlated calculations, ${ }^{26,42}$ independent of whether the latter include higher than three-body terms and whether they are evaluated harmonically or within anharmonic approximations. ${ }^{26}$ Clearly, the $\mathrm{OH}$ mode coupling in the most accurate analytical water potentials is underestimated, possibly at the level of specific coupling terms in the analytical many-body expansion.

We emphasize that the experimental data are quite robust, at least concerning $W 4$, which only relies on the dominant transitions of each spectroscopic technique. It appears that coupling among the $\mathrm{OH}$ oscillators, and thus resonant energy flow along the $\mathrm{OH}$ stretching modes, is underestimated by at least a factor of two in the most accurate analytical fits. Since the multidimensional fits are truly challenging and the investigated quantity is relatively subtle, this is not an unexpected performance, but the present experiments for the first time provide solid evidence and incentive to improve the flexibility of this particular coordinate coupling. However, in order to exclude a coincidental mismatch, it is necessary to turn to the more difficult trimer case.

\subsection{Water trimer}

For the cyclic trimer (ref. 4 and 47 and references cited therein) one has to distinguish between a $C_{3}$ symmetric aggregate (simple Hückel model) where coupling constants between the three oscillators are equal and an asymmetric coupling case where the H-bonds differ in strength. ${ }^{19}$ For the first case the solutions

$$
\left|\begin{array}{lll}
x & W & W \\
W & x & W \\
W & W & x
\end{array}\right|=0
$$

are:

$$
\begin{gathered}
x_{1}=-2 W_{1} \quad(\mathrm{~A}) \\
x_{2,3}=W \quad(\mathrm{E})
\end{gathered}
$$

Therefore the coupling constant $W$ corresponds to the splitting between the Raman and IR active levels divided by 3 .

In the latter case one obtains two different coupling constants $W_{1}, W_{2}$ and the determinant equation

$$
\left|\begin{array}{ccc}
x & W_{1} & W_{2} \\
W_{1} & x & W_{1} \\
W_{2} & W_{1} & x
\end{array}\right|=0
$$


Its solutions are:

$$
\begin{aligned}
& x_{1}=-\frac{\sqrt{8 W_{1}^{2}+W_{2}^{2}}}{2}-\frac{W_{2}}{2} \\
& x_{2}=W_{2} \\
& x_{3}=\frac{\sqrt{8 W_{1}^{2}+W_{2}^{2}}}{2}-\frac{W_{2}}{2}
\end{aligned}
$$

The splitting between the highest and lowest level is therefore $\sqrt{8 W_{1}^{2}+W_{2}^{2}}$ and the inner level is separated from the center of the multiplet by $W_{2}$.

The experimental infrared $\mathrm{OH}$ stretching spectrum of the water trimer has been studied in different environments. ${ }^{4,10,33,48,49}$ It consists of a main band and a satellite, which is often, but not always located at higher frequency. Upon deuteration, this satellite peak disappears, hinting at a possible tunneling origin. Fig. S8 in the ESI $\dagger$ discusses the phenomenological situation in comparison to the tetramer. Comparison between experiment and theory depends on whether one interprets the weak satellite in the IR as one of the near-degenerate $\mathrm{OH}$ stretching modes or not. The maximum spread of the $\mathrm{OH}$ stretching modes is either $57 \mathrm{~cm}^{-1}$ or else $\approx 42 \mathrm{~cm}^{-1}$. A more likely value is in between, if one interprets the center of gravity of the two IR bands as the proper reference. On the theoretical side, two fit variants of the three body potential yield a spread of the bound $\mathrm{OH}$ stretching modes of $50 \mathrm{vs} .29 \mathrm{~cm}^{-1}$ for the water trimer. ${ }^{50}$ Matrix isolation IR studies ${ }^{4}$ find a large sensitivity of the trimer stretching spectra and their intensities to the environment. The Ne matrix isolation value for the exciton splitting ${ }^{5}$ of $57 \mathrm{~cm}^{-1}$ is consistent with our gas phase findings, in particular considering actual matrix-shifts of $15-19 \mathrm{~cm}^{-1}$ in this case.

The non-equivalence of the three hydrogen bonds in the trimer can be included in the analysis and adds further to the uncertainty. In Table 3, we list the experimentally derived trimer coupling constants according to the symmetric $(W=W 3)$ and the asymmetric models $\left(W_{1}, W_{2}\right)$. For the regular hydrogen isotope, we distinguish between the interpretation of the main IR peak as the quasidegenerate
$\mathrm{E}$ band (case $3_{\mathrm{A}}$ ) and the interpretation of both observed peaks as the components of the $\mathrm{E}$ band despite their different intensities and large splitting (less likely case $3_{\mathrm{B}}$ ). Despite the assignment uncertainty, one can see that the $W$ coupling constants show the expected trend with deuteration (decrease) and methyl group substitution (increase), as in the case of the tetramer. When comparing to theory, we always interpret the (now equally intense and more closely spaced) IR doublet in the sense of model $3_{\mathrm{B}}$, when extracting a symmetric coupling constant $W$. The performance of harmonically predicted coupling constants is again remarkable, quite independent of the level of computation, as Table 3 shows. The coupling pattern appears to be rather robust, as expected for a transition dipole or hydrogen bond mediated interaction. This is also the case for one of the analytical WHBB (Wang/Huang/Braams/Bowman) fits to the high level $a b$ initio data, namely the variant $3 \mathrm{~b} 6$ for the three body contribution. ${ }^{50}$ On the other hand, the $3 \mathrm{~b} 5$ fit only recovers about half of the coupling. As this parametrization appears to have been used for the tetramer predictions, ${ }^{26,51}$ this may contribute to the discrepancies discussed in the previous section.

\subsection{Tentative pentamer analysis}

For the cyclic water pentamer, the absence of symmetry ${ }^{52}$ complicates the coupling analysis and several assumptions are necessary to analyze the experimental coupling pattern. We assume an averaged effective $C_{5 \mathrm{~h}}$ symmetry, instead of the puckered non-planar ring. Based on simple connectivity considerations, the nearest and next-nearest neighbor excitonic splittings are determined from the following equation:

$$
\left|\begin{array}{ccccc}
x & W_{1} & W_{2} & W_{2} & W_{1} \\
W_{1} & x & W_{1} & W_{2} & W_{2} \\
W_{2} & W_{1} & x & W_{1} & W_{2} \\
W_{2} & W_{2} & W_{1} & x & W_{1} \\
W_{1} & W_{2} & W_{2} & W_{1} & x
\end{array}\right|=0
$$

Table 3 Different theoretical values for the coupling constant $W 3=W$ for a $C_{3}$-symmetric trimer (the simple Hückel model, one level with A-symmetry

\begin{tabular}{|c|c|c|c|c|c|}
\hline Method & Variant & Electronic structure & $W / \mathrm{cm}^{-1}$ & $W_{1} / \mathrm{cm}^{-1}$ & $W_{2} / \mathrm{cm}^{-1}$ \\
\hline Harmonic $^{44}$ & BSSE-corrected & $\mathrm{MP} 2 / 6-311+\mathrm{G}(2 \mathrm{~d}, 2 \mathrm{p})$ & 17 & 19 & 13 \\
\hline Vibrational $\mathrm{CI}^{44}$ & BSSE-corrected & $\mathrm{MP} 2 / 6-311+\mathrm{G}(2 \mathrm{~d}, 2 \mathrm{p})$ & 19 & 21 & 16 \\
\hline Vibrational $\mathrm{CI}^{44}$ & With BSSE & $\mathrm{MP} 2 / 6-311+\mathrm{G}(2 \mathrm{~d}, 2 \mathrm{p})$ & 24 & 26 & 20 \\
\hline Harmonic $^{42}$ & & $\operatorname{PES}(1,2,3)$ & 11 & 13 & 7 \\
\hline Harmonic $^{42}$ & & $\operatorname{CCSD}(\mathrm{T}) / \mathrm{aug}$-cc-pVTZ & 20 & 22 & 16 \\
\hline Harmonic $^{42}$ & & CP-CCSD(T)/aug-cc-pVTZ & 18 & 20 & 14 \\
\hline Harmonic $^{42}$ & & LCCSD(T)/aug-cc-pVTZ & 15 & 16 & 11 \\
\hline Harmonic $^{50}$ & & LCCSD(T)/aug-cc-pVQZ & 21 & 23 & 17 \\
\hline Harmonic $^{50}$ & 3b5 fit & WHBB-surface & 8 & 10 & 4 \\
\hline Harmonic $^{50}$ & $3 \mathrm{~b} 6$ fit & WHBB-surface & 15 & 17 & 10 \\
\hline Harmonic $^{45}$ & & $\operatorname{CCSD}(\mathrm{T}) / \mathrm{aug}$-cc-pVDZ & 18 & 20 & 15 \\
\hline Anharmonic ${ }^{45}$ & MP2 VPT2 est. & $\operatorname{CCSD}(\mathrm{T}) / \mathrm{aug}$-cc-pVDZ & 15 & 17 & 9 \\
\hline Experiment $\mathrm{H}_{2} \mathrm{O}\left(3_{\mathrm{A}}\right)$ & $\mathrm{IR}+$ Raman & Non-Born-Oppenheimer & 14 & 14 & 14 \\
\hline Experiment $\mathrm{H}_{2} \mathrm{O}\left(3_{\mathrm{B}}\right)$ & IR + Raman & Non-Born-Oppenheimer & 17 & 20 & 9 \\
\hline Experiment $\mathrm{D}_{2} \mathrm{O}$ & IR + Raman & Non-Born-Oppenheimer & 11 & 11 & 11 \\
\hline Experiment $\mathrm{CH}_{3} \mathrm{OH}^{19}$ & IR + Raman & Non-Born-Oppenheimer & 20 & 21 & 17 \\
\hline Experiment $\mathrm{CH}_{3} \mathrm{OD}^{19}$ & IR + Raman & Non-Born-Oppenheimer & 13 & 14 & 11 \\
\hline
\end{tabular}
(Raman active) and two degenerate levels of E-symmetry (IR-active)) and coupling constants $W_{1}$ and $W_{2}$ for the more complex case where the hydrogen bonds differ in strength (asymmetric coupling). Experimental trimer coupling constants are less certain than tetramer constants because of the reduced symmetry, the potentially large tunneling splitting, and the intensity difference of the two observed IR-active bands 
The solutions to this determinant equation $x_{i}$ relative to the center of gravity of the multiplet are (with $g=\frac{1}{g}-1=$ $(\sqrt{5}-1) / 2$ as the golden ratio):

$$
\begin{array}{cc}
x_{1}=-2 W_{1}-W_{2} \quad\left(\mathrm{~A}^{\prime}\right) \\
x_{2,3}=-g W_{1}+W_{2} / g \quad\left(\mathrm{E}_{1}{ }^{\prime}\right) \\
x_{4,5}=W_{1} / g-g W_{2} \quad\left(\mathrm{E}_{2}{ }^{\prime}\right)
\end{array}
$$

The splitting between the dominant IR band $\left(\mathrm{E}_{1}{ }^{\prime}\right)$ and the dominant Raman band $\left(\mathrm{A}^{\prime}\right)$ is thus $(2-g) W_{1}+(3+g) W_{2}$, whereas the splitting between the two Raman active bands is $(3+g) W_{1}+(2-g) W_{2}$. In a puckered ring, the degenerate states will be further split by a small amount. Because the highest frequency transition is not predicted to be strong in either IR or Raman spectra, it is desirable to have a linear combination of coupling constants $W 5$ which does not depend on its assignment. This is given by $W 5=W_{1}+\frac{\sqrt{5}+1}{\sqrt{5}-1} \cdot W_{2}$ which we therefore use as a robust measure for pentameric exciton coupling. As in the case of $W 4, W 5$ corresponds to the nearest neighbor coupling $W_{1}$ if more distant couplings are neglected. The experimental error in $W 5$ is less than $\pm 3 \mathrm{~cm}^{-1}$, if we assume a conservative error in the IR/Raman-splitting of $4 \mathrm{~cm}^{-1}$.

Turning now to the experimental spectra, the dominant IR/Raman water pentamer signals (Fig. 2 and 3) are closer together than the tetramer peaks, despite a reduced ring strain and enhanced cooperativity in the cyclic hydrogen bond pattern. Only the deuterated water spectra offer an assignment of all three bands, if the band at $2500 \mathrm{~cm}^{-1}$ marked $>3 \mathrm{~b}$ is tentatively interpreted as the transition to $\mathrm{E}_{2}{ }^{\prime}$. One then obtains $W_{1}=14 \mathrm{~cm}^{-1}$ and $W_{2}=4 \mathrm{~cm}^{-1}$, which may be compared with the corresponding tetramer values of 17 and $5 \mathrm{~cm}^{-1}$, respectively. The coupling parameter $W 5$, which only depends on the strong IR and Raman features, is also systematically smaller than for the theoretical harmonic predictions, even at levels that were shown to be quite successful for tetramers and trimers (see Table 4). We think that the explanation is of an anharmonic nature. For this, the region around $3200 \mathrm{~cm}^{-1}$ $\left(2400 \mathrm{~cm}^{-1}\right.$ for $\mathrm{D}_{2} \mathrm{O}$ ) must be addressed. ${ }^{34}$ Blue-shifted to the Raman transitions of the first monomer $\mathrm{OH}$ bending overtone (10), there is a polarized signal $(>30)$ near $3214 \mathrm{~cm}^{-1}$ which has an intensity evolution typical for a mix of ring tetramers and pentamers. This is illustrated in Fig. 4. Its IR counterparts are weak and somewhat shifted (see Fig. 2 and 3), ${ }^{39}$ supporting a dominant ring cluster contribution. In Ne matrices, a blueshifted $^{53}$ trimer bending overtone has been reported ${ }^{5}$ at $3190 \mathrm{~cm}^{-1}$. Normally, one would expect much less Raman intensity for such a cluster transition, due to the dominance of monomers in the expansions. Furthermore, a coincidence of tetramer and pentamer signals is unlikely in an unperturbed picture. ${ }^{43}$ The progressively smaller energy gap between the $\mathrm{OH}$ stretching fundamentals $(4 \mathrm{~b}, 5 \mathrm{~b})$ and the $\mathrm{OH}$ bending overtone $(>3 \mathrm{o})$ explains both anomalies by an increasing extent of Fermi resonance between the two states (see Fig. 1), relative to the trimer. The pentamer may have a somewhat higher bending overtone visibility and experience a reduced overtone frequency due to this anharmonic interaction. That is particularly true for the highest frequency concerted bending modes, which are expected to couple most to the Raman active concerted $\mathrm{OH}$ stretching modes (Fig. 1).

As a consequence, the latter are shifted to higher frequency, more so in the pentamer than in the tetramer. Indeed, simple model calculations show that the energy coincidence is close in the pentamer. This is illustrated in Table 5, which uses published density functional data ${ }^{43}$ to predict the energy coincidence between the experimental stretching fundamental $n \mathrm{~b}$ and the anharmonic bend overtones. The latter are estimated from the experimental monomer bend overtone and the calculated complexation shift in the fundamental region. Fermi resonance is more likely if the depolarization ratio is low (like in the

Table 4 Different theoretical results for the nearest neighbor $\left(W_{1}\right)$ and distant $\left(W_{2}\right)$ coupling parameters for the simple model of a $C_{5 h}$ symmetric water pentamer. Since the minimum structure of the pentamer is not $C_{5 \mathrm{~h}}$ symmetric and therefore exhibits no degeneracies, the mean values of the two IR active (corresponding to $E_{1}{ }^{\prime}$ ) and the highest lying two Raman active (corresponding to $E_{2}{ }^{\prime}$ ) bands have been used for the calculation of the coupling

\begin{tabular}{|c|c|c|c|c|c|}
\hline Method & Variant & Electronic structure & $W_{1} / \mathrm{cm}^{-1}$ & $W_{2} / \mathrm{cm}^{-1}$ & $W 5=W_{1}+\frac{\sqrt{5}+1}{\sqrt{5}-1} \cdot W_{2} / \mathrm{cm}^{-1}$ \\
\hline Harmonic $^{52}$ & & MP2/aug-cc-pVDZ & 35 & 9 & 58 \\
\hline Harmonic $^{43}$ & Scaled & B3LYP/d-aug-cc-pVDZ & 34 & 10 & 60 \\
\hline Harmonic $^{44}$ & BSSE-corrected & MP2/6-311+G(2d,2p) & 26 & 7 & 45 \\
\hline Vibrational $\mathrm{CI}^{44}$ & BSSE-corrected & $\mathrm{MP} 2 / 6-311+\mathrm{G}(2 \mathrm{~d}, 2 \mathrm{p})$ & 26 & 6 & 42 \\
\hline Vibrational $\mathrm{CI}^{44}$ & With BSSE & $\mathrm{MP} 2 / 6-311+\mathrm{G}(2 \mathrm{~d}, 2 \mathrm{p})$ & 43 & 34 & 133 \\
\hline Harmonic $^{26}$ & Full-dimensional & WHBB & 14 & 0 & 13 \\
\hline Harmonic $^{26}$ & LMon- $\beta_{\text {dimer }}$ & WHBB & 14 & 0 & 14 \\
\hline Anharmonic $^{26}$ & LMon- $\beta_{\text {dimer }}$ & WHBB & 15 & 0 & 14 \\
\hline Harmonic $^{45}$ & & $\operatorname{CCSD}(\mathrm{T}) /$ aug-cc-pVDZ & 30 & 9 & 53 \\
\hline Anharmonic $^{45}$ & MP2 VPT2 est. & $\operatorname{CCSD}(\mathrm{T}) /$ aug-cc-pVDZ & 36 & 21 & 90 \\
\hline Experiment $\mathrm{H}_{2} \mathrm{O}$ & IR + Raman & Non-Born-Oppenheimer & - & - & 33 \\
\hline Harmonic $^{52} \mathrm{D}_{2} \mathrm{O}$ & & MP2/aug-cc-pVDZ & 24 & 8 & 44 \\
\hline Experiment $\mathrm{D}_{2} \mathrm{O}$ & IR + Raman & Non-Born-Oppenheimer & 14 & 4 & 25 \\
\hline
\end{tabular}
constants. The combination $W 5=W_{1}+\frac{\sqrt{5}+1}{\sqrt{5}-1} \cdot W_{2}$ in the last column does not depend on the uncertain assignment of the weakly Raman active transition $\mathrm{E}_{2}{ }^{\prime}$ and is therefore most reliable 


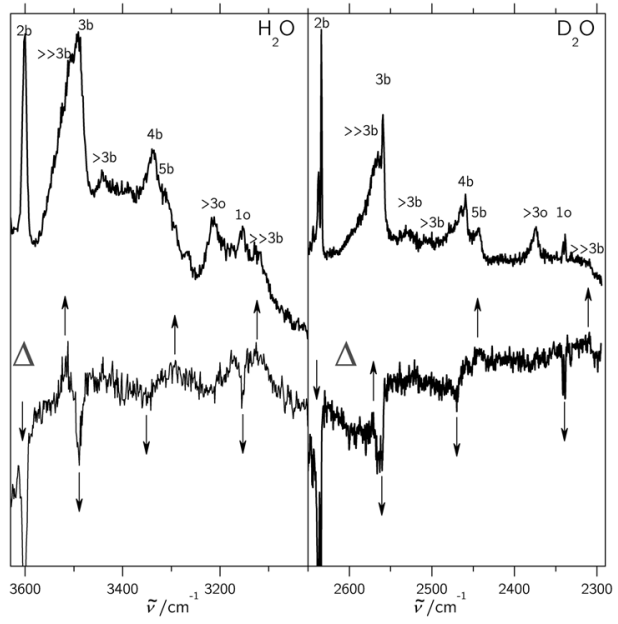

Fig. 4 Difference spectra $\Delta$ (bottom traces) showing that the $>30$ band (top traces) in the jet expansions of $\mathrm{H}_{2} \mathrm{O}$ (left) and $\mathrm{D}_{2} \mathrm{O}$ (right) has dominant contributions from cluster sizes 4 and 5 and that bands marked $\gg 3 \mathrm{~b}$ have hexamer and larger contributions. By subtracting related spectra with decreasing cluster content from each other after scaling in such a way that the $>30$ band cancels, negative peaks emerge for cluster sizes smaller than $>30$ and positive peaks result from cluster sizes larger than $>30$.

Table 5 Calculated ring cluster bending wavenumbers $\omega_{n}^{\text {bend }}$ and Raman depolarization ratios $\rho$ are used to estimate the gap $\Delta(\mathrm{b}-\mathrm{O})=\nu(n \mathrm{~b})-$ $\left(\nu(10)+2\left(\omega_{n}^{\text {bend }}-\omega_{1}^{\text {bend }}\right)\right)$ between the lowest frequency bonded $\mathrm{OH}$ stretching fundamental $n b$ and the overtones of the bending fundamentals

\begin{tabular}{llll}
\hline \multirow{2}{*}{ Cluster size } & \multicolumn{3}{l}{ B3LYP/d-aug-cc-pVDZ } \\
\cline { 2 - 3 }$\omega_{n}^{\text {bend }} / \mathrm{cm}^{-1}$ & $\rho$ & $\Delta(\mathrm{b}-\mathrm{o}) / \mathrm{cm}^{-1}$ \\
\hline 1 & 1622 & 0.68 & 504 \\
3 & & & \\
& 1658 & 0.39 & 266 \\
& 1634 & 0.64 & 314 \\
& 1631 & 0.75 & 320 \\
& & & \\
& 1681 & 0.25 & 63 \\
& 1651 & 0.75 & 123 \\
& 1636 & 0.75 & 153 \\
5 & & & \\
& 1689 & 0.17 & 23 \\
& 1681 & 0.05 & 39 \\
1660 & 0.74 & 81 \\
1652 & 0.62 & 97 \\
& 1640 & 0.73 & 121
\end{tabular}

lowest frequency $\mathrm{OH}$ stretching fundamental) and the gap $\Delta(\mathrm{b}-\mathrm{o})$ is small. One can conclude that the Raman active pentamer vibration $5 \mathrm{~b}$ is most likely to undergo strong Fermi resonance with a bending overtone 50. The two highest frequency bending modes indeed have the smallest depolarization ratios and the smallest gaps. This explains the apparently small pentamer $\mathrm{OH}$ stretch excitonic coupling in a qualitative way. We estimate $20-50 \mathrm{~cm}^{-1}$ for the order of magnitude of the Fermi resonance coupling parameter (smaller in the $\mathrm{D}_{2} \mathrm{O}$ case), but the multidimensional nature of the coupling precludes a firm experimentally derived statement. The close vicinity of the bending overtone is also responsible for the short predicted energy redistribution lifetime ${ }^{29}$ for the tetramer after Raman-active $\mathrm{OH}$ stretch excitation. As has been pointed out before,${ }^{35}$ inclusion of such anharmonic interactions is crucial for a correct modeling and the present experimental data can provide valuable constraints. While there are numerous vibrational predictions for water clusters in the literature, few of them include Raman intensities ${ }^{43}$ or anharmonic effects. ${ }^{35}$ It is hoped that anharmonic calculations including Raman intensities will be carried out in the future to compare more accurately with the present experimental spectra.

\subsection{Water hexamer}

For the water hexamer, our Raman spectra provide polarized band evidence above $3100 \mathrm{~cm}^{-1}$ and $3500 \mathrm{~cm}^{-1}$ ( $\gg 3 \mathrm{~b}$, see also Fig. 4), but the lack of size-selectivity ${ }^{54}$ does not allow for an unambiguous assignment. A ring structure can be excluded based on the comparison with He droplet spectra $^{33}$ and a dominant book isomer based on theoretical predictions. ${ }^{35}$ The stable prism and cage structures ${ }^{12}$ are both reasonably consistent with our Raman spectra ${ }^{43}$ with a strong signal above $3100 \mathrm{~cm}^{-1}\left(2300 \mathrm{~cm}^{-1}\right.$ for $\left.\mathrm{D}_{2} \mathrm{O}\right)$ and a second pile-up of Raman intensity around the trimer band. Weaker Raman signals predicted between the dominant trimer and tetramer bands are consistent with our spectra, but they overlap with $n=4$, 5 bands.

\section{Conclusions}

The first spontaneous Raman scattering spectra of small water clusters offer quantitative insights into the coherent energy hopping of localized $\mathrm{OH}$ stretching excitations from one molecule to another, when compared to existing IR spectra. This is related to the non-coincidence effect, ${ }^{55}$ which can be spectrally resolved in the case of cold clusters. ${ }^{23}$ Table 6 summarizes the robust $\mathrm{OH}$ and $\mathrm{OD}$ oscillator coupling constants for the water trimer (W3), the tetramer (W4) and the pentamer (W5). The deuteration and methylation ${ }^{19}$ effects are systematic and expected. The ring size dependence is unusually weak beyond the tetramer, which we explain by the growing importance of stretch-bend Fermi resonance for the symmetric modes. Effects of intra-monomer coupling should be smaller in the case of cyclic water clusters, because the strong hydrogen bonds decouple the free and the bound $\mathrm{OH}$ stretching modes quite effectively.

The excitonic coupling constants obtained in this experimental study for cyclic water clusters provide valuable constraints on harmonic, ${ }^{20}$ anharmonic ${ }^{26,44}$ and empirical ${ }^{46}$ models for the

Table 6 Robust experimental $\mathrm{OH}$ oscillator coupling parameters $W n$ for $n$-mers of $\mathrm{M}=\mathrm{H}_{2} \mathrm{O}, \mathrm{D}_{2} \mathrm{O}, \mathrm{CH}_{3} \mathrm{OH}$. The experimental error due to Raman calibration and finite spectral band widths is less than $2 \mathrm{~cm}^{-1}$ for $W 4$ and less than $3 \mathrm{~cm}^{-1}$ for $W 5$

\begin{tabular}{llll}
\hline $\mathrm{M}$ & $W 3 / \mathrm{cm}^{-1}$ & $W 4 / \mathrm{cm}^{-1}$ & $W 5 / \mathrm{cm}^{-1}$ \\
\hline $\mathrm{H}_{2} \mathrm{O}$ & $14-17$ & 34 & 33 \\
$\mathrm{D}_{2} \mathrm{O}$ & 11 & 22 & 25 \\
$\mathrm{CH}_{3} \mathrm{OH}^{19}$ & 20 & 40 &
\end{tabular}


vibrational dynamics in hydrogen-bonded systems and water in particular. In a somewhat model-dependent way, they can be interpreted within a time-dependent framework. Already for the trimer, the splittings are consistent with a drop of any local excitation to $1 / e$ within about $0.3 \mathrm{ps}^{19,27}$ For tetramers and pentamers, the coherent transfer to neighboring modes will be even faster. In an extended network such as liquid water, this decay becomes irreversible and it qualitatively explains the ultrafast energy flow observed in fs-experiments, ${ }^{22,36,56}$ even without having to invoke Fermi resonance with the bending overtone and other relaxation channels. The latter mechanisms will follow, in particular for cluster sizes beyond the trimer, and ultimately dissipate the energy into low frequency modes. ${ }^{29}$ However, the primary step seems to be explainable in a harmonic picture ${ }^{57}$ of mode couplings and this is consistent with the present obervation that harmonically determined coupling constants agree quite well with experiment up to the tetramer. Water potentials used for the modeling of fs dynamics in water $^{8,26,50}$ should thus be tested for their ability to predict the IR/Raman splitting observed in water clusters in this work. This hydrogen bond-induced excitonic splitting is a subtle, yet crucial ingredient for multidimensional cluster potential energy surfaces, in particular for the smallest clusters of the most important molecule on earth.

\section{Acknowledgements}

This project has been funded by the DFG project Su 121/2. We also thank the Fonds der Chemischen Industrie and the DFG research training group 782 (www.pcgg.de) for support, N. Lüttschwager for developing the heated nozzle setup which was used to vary the cluster concentrations, and S. Montero for valuable early advice on Raman jet spectroscopy.

\section{References}

1 H. J. Bakker and J. L. Skinner, Chem. Rev., 2010, 110, 1498-1517.

2 F. N. Keutsch and R. J. Saykally, Proc. Natl. Acad. Sci. U. S. A., 2001, 98, 10533-10540.

3 U. Buck and F. Huisken, Chem. Rev., 2000, 100, 3863-3890.

4 J. Ceponkus, P. Uvdal and B. Nelander, J. Chem. Phys., 2011, 134, 064309.

5 B. Tremblay, B. Madebène, M. Alikhani and J. Perchard, Chem. Phys., 2010, 378, 27-36.

6 C. C. Pradzynski, R. M. Forck, T. Zeuch, P. Slavícek and U. Buck, Science, 2012, 337, 1529-1532.

7 S. Y. Fredericks, J. M. Pedulla, K. D. Jordan and T. S. Zwier, Theor. Chem. Acc., 1997, 96, 51-55.

8 G. C. Groenenboom, E. M. Mas, R. Bukowski, K. Szalewicz, P. E. S. Wormer and A. van der Avoird, Phys. Rev. Lett., 2000, 84, 4072-4075.

9 L. M. Goss, S. W. Sharpe, T. A. Blake, V. Vaida and J. W. Brault, J. Phys. Chem. A, 1999, 103, 8620-8624.
10 D. J. Nesbitt, T. Häber and M. A. Suhm, Faraday Discuss., 2001, 118, 305-306.

11 A. Moudens, R. Georges, M. Goubet, J. Makarewicz and S. E. Lokshtanov, J. Chem. Phys., 2009, 131, 204312.

12 C. Pérez, M. T. Muckle, D. P. Zaleski, N. A. Seifert, B. Temelso, G. C. Shields, Z. Kisiel and B. H. Pate, Science, 2012, 336, 897-901.

13 S. Wuelfert, D. Herren and S. Leutwyler, J. Chem. Phys., 1987, 86, 3751-3753.

14 B. Nelander, J. Chem. Phys., 1988, 88, 5254-5256.

15 S. Wuelfert, D. Herren and S. Leutwyler, J. Chem. Phys., 1988, 88, 5256-5257.

16 T. I. C. Jansen, B. M. Auer, M. Yang and J. L. Skinner, J. Chem. Phys., 2010, 132, 224503.

17 U. Buck, I. Ettischer, M. Melzer, V. Buch and J. Sadlej, Phys. Rev. Lett., 1998, 80, 2578-2581.

18 J. B. Paul, C. P. Collier, R. J. Saykally, J. J. Scherer and A. O'Keefe, J. Phys. Chem. A, 1997, 101, 5211-5214.

19 R. W. Larsen, P. Zielke and M. A. Suhm, J. Chem. Phys., 2007, 126, 194307.

20 G. Firanescu, D. Luckhaus and R. Signorell, J. Chem. Phys., 2008, 128, 184301.

21 M. L. Cowan, B. D. Bruner, N. Huse, J. R. Dwyer, B. Chugh, E. T. J. Nibbering, T. Elsaesser and R. J. D. Miller, Nature, 2005, 434, 199-202.

22 H. Torii, J. Phys. Chem. A, 2006, 110, 9469-9477.

23 C. J. Tainter, Y. Ni, L. Shi and J. L. Skinner, J. Phys. Chem. Lett., 2013, 4, 12-17.

24 S. Garrett-Roe and P. Hamm, Phys. Chem. Chem. Phys., 2010, 12, 11263-11266.

25 A. Paarmann, T. Hayashi, S. Mukamel and R. J. D. Miller, J. Chem. Phys., 2008, 128, 191103.

26 Y. Wang and J. M. Bowman, J. Chem. Phys., 2012, 136, 144113.

27 M. A. Suhm, Adv. Chem. Phys., 2009, 142, 1-57.

28 C. M. Tanner, M. Quack and D. Schmidiger, J. Phys. Chem. A, 2013, 117, 10105-10118.

29 Y. Niu, R. Pang, C. Y. Zhu, M. Hayashi, Y. Fujimura, S. H. Lin and Y. R. Shen, Chem. Phys. Lett., 2013, 586, 153-158.

30 G. Avila, J. M. Fernández, G. Tejeda and S. Montero, J. Mol. Spectrosc., 2004, 228, 38-65.

31 A. van der Avoird and K. Szalewicz, J. Chem. Phys., 2008, 128, 014302.

32 K. Kuyanov-Prozument, M. Y. Choi and A. F. Vilesov, J. Chem. Phys., 2010, 132, 014304.

33 C. J. Burnham, S. S. Xantheas, M. A. Miller, B. E. Applegate and R. E. Miller, J. Chem. Phys., 2002, 117, 1109-1122.

34 C. Steinbach, P. Andersson, M. Melzer, J. K. Kazimirski, U. Buck and V. Buch, Phys. Chem. Chem. Phys., 2004, 6, 3320-3324.

35 Y. Wang and J. M. Bowman, J. Phys. Chem. Lett., 2013, 4, 1104-1108.

36 C. J. Tainter and J. L. Skinner, J. Chem. Phys., 2012, 137, 104304.

37 G. Tejeda, M. Fernández and S. Montero, AIP Conf. Proc., 2012, 1501, 1305. 
38 G. E. Walrafen, J. Chem. Phys., 1964, 40, 3249-3256.

39 J. B. Paul, R. A. Provencal, C. Chapo, A. Petterson and R. J. Saykally, J. Chem. Phys., 1998, 109, 10201-10206.

40 M. A. Suhm, Science, 2004, 304, 823.

41 M. Y. Tretyakov, E. A. Serov, M. A. Koshelev, V. V. Parshin and A. F. Krupnov, Phys. Rev. Lett., 2013, 110, 093001.

42 Y. Wang, B. C. Shepler, B. J. Braams and J. M. Bowman, J. Chem. Phys., 2009, 131, 054511.

43 H. Cybulski and J. Sadlej, Chem. Phys., 2007, 342, 163-172.

44 Y. Watanabe, S. Maeda and K. Ohno, J. Chem. Phys., 2008, 129, 074315.

45 E. Miliordos, E. Aprà and S. S. Xantheas, J. Chem. Phys., 2013, 139, 114302.

46 V. Buch, S. Bauerecker, J. P. Devlin, U. Buck and J. K. Kazimirski, Int. Rev. Phys. Chem., 2004, 23, 375-433.

47 T. Salmi, E. Sälli and L. Halonen, J. Phys. Chem. A, 2012, 116, 5368-5374.

48 J. Ceponkus, G. Karlström and B. Nelander, J. Phys. Chem. A, 2005, 109, 7859-7864.
49 J. Ceponkus, P. Uvdal and B. Nelander, J. Phys. Chem. A, 2012, 116, 4842-4850.

50 Y. Wang, X. Huang, B. C. Shepler, B. J. Braams and J. M. Bowman, J. Chem. Phys., 2011, 134, 094509.

51 Y. Wang and J. M. Bowman, J. Chem. Phys., 2011, 134, 154510.

52 S. Graf, W. Mohr and S. Leutwyler, J. Chem. Phys., 1999, 110, 7893-7908.

53 J. B. Paul, R. A. Provencal, C. Chapo, K. Roth, R. Casaes and R. J. Saykally, J. Phys. Chem. A, 1999, 103, 2972-2974.

54 E. G. Diken, W. H. Robertson and M. A. Johnson, J. Phys. Chem. A, 2004, 108, 64-68.

55 M. Yang and J. L. Skinner, Phys. Chem. Chem. Phys., 2010, 12, 982-991.

56 J. Lindner, P. Vöhringer, M. S. Pshenichnikov, D. Cringus, D. A. Wiersma and M. Mostovoy, Chem. Phys. Lett., 2006, 421, 329-333.

57 J. A. Poulsen, G. Nyman and S. Nordholm, J. Phys. Chem. A, 2003, 107, 8420-8428. 